\title{
Interventional nuclear medicine
}

\author{
V. Ralph McCready • Sabina Dizdarevic • Mark Aplin
}

Published online: 3 May 2013

(C) Springer-Verlag Berlin Heidelberg 2013

Interventional radiology is well established and has been described as the use of minimal invasive surgery performed with the aid of simultaneous radiological imaging. It is surprising that interventional nuclear medicine has not been developed. Interventional nuclear medicine would employ a similar approach where, with the aid of a simple intervention radionuclide, treatment can be provided for benign and malignant pathology. The main difference between interventional radiology and nuclear medicine is that while the former employs an anatomical approach with radiofrequency, ultrasound and cryotherapy ablating a volume which is larger than the lesion, the nuclear medicine approach can use some aspect of physiology to locate the radiopharmaceutical within the target volume and especially any microscopic extension of the pathology.

Probably the first attempt at interventional nuclear medicine involved the use of ${ }^{32} \mathrm{P}$-labelled colloid. Order and colleagues in the USA injected the ${ }^{32} \mathrm{P}$ colloid into pancreatic carcinoma, a disease which is resistant to external beam radiation and many forms of chemotherapy [1]. Westlin and colleagues also treated patients with pancreatic tumours with ${ }^{32} \mathrm{P}$ colloid [2]. Firusian and Dempke used the same radiopharmaceutical to treat 17 patients with refractory solid tumours or solitary metastases [3]. Complete remission was seen in 7 patients $(41 \%)$, and partial remission in $29 \%$ (5 patients). ${ }^{32} \mathrm{P}$ gives very high radiation doses not possible with external beam radiotherapy, but the fact that these studies have not been followed up could be because the high interstitial tumour pressure means that the colloid spread is very limited and the high injection pressure required, at least in pancreatic tumours, makes the technique somewhat dangerous for the operator [4]. The difficulty in injecting the radiopharmaceutical is probably due to the colloid stabilizer.

\footnotetext{
V. R. McCready $(\bowtie) \cdot S$. Dizdarevic $\cdot$ M. Aplin

Department of Nuclear Medicine, Royal Sussex County Hospital, Brighton, East Sussex BN2 5BE, UK

e-mail: nukdoctor@hotmail.com
}

In this issue of the journal, Botta et al. report the development of the concept of using a limited diffusion radiopharmaceutical with multiple injections for the treatment of prostate cancer [5]. The use of ${ }^{90} \mathrm{Y}$ avidin has been suggested as a method of overcoming the problem of the migration of ${ }^{125} \mathrm{I}$ seeds used in brachytherapy. Presumably the same approach could be used in other superficial tumours, although the more convenient approach of injecting a diffusible targeted radiopharmaceutical as outlined below would be preferable.

It is difficult to administer high tumoricidal doses of radiation by intravenous injection of a labelled radiopharmaceutical since tumour uptake is limited by its blood flow. This can be partially overcome by administering the activity via a blood vessel supplying the pathology. It has been shown that in hepatic neuroendocrine tumours there is a considerable improvement in the uptake of ${ }^{68} \mathrm{Ga}$ DOTATOC following intraarterial injection compared with intravenous injection [6]. Similarly intraarterial administration can be used to enhance the therapeutic effect of ${ }^{90} \mathrm{Y} /{ }^{177} \mathrm{Lu}$ DOTATOC in suitable accessible tumours [7]. Using a feeding artery in a patient with an unresectable meningioma it has been possible to administer a dose of 500 Gy with 5 GBq DOTA-TATE (Baum R.P. 2013, personal communication). In studies using microspheres to treat hepatic carcinoma, limitations of the intraarterial approach with a nonspecific radiopharmaceutical have been seen with the irradiation of normal hepatic tissue and the shunting of microspheres through the liver to the lungs. Suitable selection of patients can minimize these side effects, but overall the outcomes of treatment with ${ }^{90} \mathrm{Y}$ microspheres in terms of tumour response and survival are limited $[8,9]$.

The optimum treatment for benign and malignant tumours that are superficial and accessible is the direct percutaneous injection of a radionuclide-labelled carrier into the pathology (PErcutaneous injection of Radionuclide THerapyPERTH). Using this approach accurately calculated optimal high radiation doses not achievable with external beam radiotherapy can be administered without irradiation of normal 
tissues. For example, $671 \mathrm{MBq}{ }^{111}$ In octreotide retained in a 20$\mathrm{cm}^{3}$ tumour would give a dose of $100 \mathrm{~Gy}$ and $44 \mathrm{MBq}{ }^{90} \mathrm{Y}$ octreotide would give the same dose. The ideal carrier is tumourspecific, but if it is not retained in the tumour the activity lost from the lesion would not produce a significant whole-body dose. For example, in the case of ${ }^{111}$ In octreotide the activity that would be required to give 100 Gy to a tumour by direct injection would give a whole-body dose of only $37 \mathrm{mGy}$ if there was complete loss from the tumour, indicating a high level of safety.

In the search for suitable carriers for direct injection, experimental model tumours have shown that most of the presently commercially available radiopharmaceuticals would not be suitable. Focal injections of ${ }^{32} \mathrm{P}$ colloids result in minimal spread and subsequent loss from tumours via the vascular system to the reticuloendothelial system [10]. Similarly, ${ }^{90} \mathrm{Y}$ microspheres and radioiodine-labelled lipiodol do not diffuse from the point of injection. In experimental models so-called tumour-seeking radiopharmaceuticals such as DMSA(V) and gallium citrate have shown limited long-term retention and there is spread outside the target lesion. Exametazime (HMPAO) shows high tissue retention in proportion to tissue perfusion. Following intravenous injection increased uptake in lung tumours has been demonstrated [11] but the therapeutic possibility of ${ }^{188} \mathrm{Re}$ exametazime as a suitable carrier for direct intratumoral injection has not been explored.

The possible model for the use of intralesional injections of radionuclides would be thyroid toxic nodules. The nodules are superficial and easily accessible. Iodide is retained in thyroid tissue following oral or intravenous administration with high efficiency. Direct injection of ${ }^{131} \mathrm{I}$ into a toxic nodule would require only $123 \mathrm{MBq}$ to give a radiation dose of $400 \mathrm{~Gy}$ to a $10-\mathrm{ml}$ nodule. This compares with the recommended oral administration of 500-800 MBq [12]. The use of this approach would limit the disadvantages of oral administration which include the irradiation of normal thyroid tissue resulting in long-term hypothyroidism, and unnecessary irradiation of other organs. There is also a small but measurable risk of inducing cancer both in the thyroid and elsewhere $[13,14]$. A clinical trial would be required to determine the optimum radiation dose for nodule ablation since currently doses delivered by oral activities cannot be directly measured. The injection volume required to uniformly diffuse to the edges of a volume measured by ultrasound without extranodular spill has also to be determined.

For malignant and some benign tumours the current most likely candidate for intratumoral therapy would be a somatostatin analogue. Unlike antibodies, somatostatin analogues diffuse through the tissue [15]. There are somatostatin receptors in a wide variety of tumours which are accessible by percutaneous injection [16]. These include medullary carcinoma, some neuroblastomas, phaeochromocytomas, carcinoid, small-cell carcinomas and breast carcinomas.
A further possible scenario for percutaneous radionuclide direct injection is the ablation of primary breast carcinoma and microscopic extension prior to surgery by injection of a ${ }^{111}$ In-labelled or ${ }^{90}$ Y-labelled somatostatin analogue such as octreotide. An activity of only $44 \mathrm{MBq}{ }^{90} \mathrm{Y}$ somatostatin analogue would deliver $100 \mathrm{~Gy}$ to a $20-\mathrm{cm}^{3}$ tumour. Assuming that the radiopharmaceutical would be $100 \%$ retained, the administration of concomitant chemotherapy should pose no problem since there should be no irradiation of the bone marrow.

A study of the use of a directly injected radiopharmaceutical has demonstrated encouraging results confirming that the technique is possible and outcomes improve. ${ }^{90} \mathrm{Y} /{ }^{177} \mathrm{Lu} /{ }^{213} \mathrm{Bi}-$ labelled peptide substance $\mathrm{P}$ has been injected locally into malignant glial brain tumours. The radiopharmaceutical distributed according to the tumour geometry and following resection widespread necrosis was seen $[17,18]$.

The question arises as to why directly injected radiopharmaceuticals have not been implemented more widely already. Unlike radiological interventionists, nuclear physicians are perhaps more hesitant to use an intralesional injection. There is a view that because cancer is a systemic disease, this approach would not be appropriate. However, the increasing use of anatomically based radiological interventional techniques demonstrates the need for localized ablative therapy. Interventional nuclear medicine has many potential advantages. The use of a radiopharmaceutical targeted to a specific lesion should be more effective and less traumatic than current anatomically based radiological interventional techniques. The localization of the radiopharmaceutical enables high doses to be delivered to the pathology in a safe manner reducing unnecessary irradiation of normal tissues. The low activities required to give high doses offer the potential to improve radiation protection for staff while being cost effective. Of course, for interventional nuclear medicine to be successful, further research is required into developing novel tumour-specific carriers, and into determining the optimum radionuclide or combination of radionuclides, the diffusion characteristics of tumours and the optimal doses for lesion ablation. An example of the importance of using the correct dose is seen in the study in this issue of the journal by Garin et al. [19] who found a good correlation between tumour dose and response in patients with hepatocellular carcinoma treated with ${ }^{90}$ Y-loaded glass microspheres.

In the paper in this issue of the journal, Botta et al. [5] raise and discuss the issue of comparing the doses delivered from different radionuclides with different emissions and half-lives in relation to their biological effectiveness. The concept of biological effective dose has recently been reviewed by Fowler [20]. Until now it has been applied more to external beam radiotherapy, but it will also be important in the development of interventional nuclear medicine techniques especially when applied to the combination 
of external beam radiotherapy, chemotherapy and irradiation by locally injected radiopharmaceuticals.

Unfortunately, even when interventional nuclear medicine involves using an already licensed radiopharmaceutical in a novel way an expensive clinical trial is required. Hopefully the potential clinical and economic advantages of this approach will encourage investment in this new aspect of nuclear medicine.

\section{References}

1. Order SE, Siegel JA, Principato R, Zeiger LS, Johnson E, Lang P, et al. Preliminary experience of infusional brachytherapy using colloidal 32P. Ann Acad Med Singapore. 1996;25(3):347-51.

2. Westlin JE, Andersson-Forsman C, Garske U, Linne T, Aas M, Glimelius B, et al. Objective responses after fractionated infusional brachytherapy of unresectable pancreatic adenocarcinomas. Cancer. 1997;80(12 Suppl):2743-8.

3. Firusian N, Dempke W. An early phase II study of intratumoral P32 chromic phosphate injection therapy for patients with refractory solid tumors and solitary metastases 2. Cancer. 1999;85(4):980-7.

4. McCready VR, Heriot AG, Masoomi M, Britton A, Glees PJ, Knight M, et al. P32 interstitial therapy for unresectable carcinoma of the pancreas. J Nucl Med. 1999;40(5):984. Abstract.

5. Botta F, Cremonesi M, Ferrari ME, Amato E, Guerriero F, Vavassori A, et al. Investigation of $90 \mathrm{Y}$-avidin for prostate cancer brachytherapy: a dosimetric model for phase I-II clinical study. Eur J Nucl Med Mol Imaging. 2013. doi:10.1007/s00259-013-2383-1.

6. Kratochwil C, Giesel FL, Lopez-Benitez R, Schimpfky N, Kunze $\mathrm{K}$, Eisenhut $\mathrm{M}$, et al. Intraindividual comparison of selective arterial versus venous $68 \mathrm{Ga}$-DOTATOC PET/CT in patients with gastroenteropancreatic neuroendocrine tumors. Clin Cancer Res. 2010;16(10):2899-905.

7. Kratochwil C, Lopez-Benitez R, Mier W, Haufe S, Isermann B, Kauczor HU, et al. Hepatic arterial infusion enhances DOTATOC radiopeptide therapy in patients with neuroendocrine liver metastases. Endocr Relat Cancer. 2011;18(5):595-602.

8. Hilgard P, Hamami M, Fouly AE, Scherag A, Muller S, Ertle J, et al. Radioembolization with yttrium-90 glass microspheres in hepatocellular carcinoma: European experience on safety and longterm survival. Hepatology. 2010;52(5):1741-9.
9. Salem R, Lewandowski RJ, Mulcahy MF, Riaz A, Ryu RK, Ibrahim S, et al. Radioembolization for hepatocellular carcinoma using yttrium-90 microspheres: a comprehensive report of longterm outcomes. Gastroenterology. 2010;138(1):52-64.

10. Hammersley PAG, Vini L, McCready VR. Intra-tumoural infusion of sources for radiotherapy of solid tumours. J Nucl Med. 1997;38(5SS):830. Abstract.

11. Rowell NP, McCready VR, Tait D, Flower MA, Cronin B, Adams GE, et al. Technetium-99m HMPAO and SPECT in the assessment of blood flow in human lung tumours. Br J Cancer. 1989;59(1):135-41.

12. Royal College of Physicians. Radioiodine in the management of benign thyroid disease: clinical guidelines. Report of a working party. London: Royal College of Physicians; 2007.

13. Metso S, Auvinen A, Huhtala H, Salmi J, Oksala H, Jaatinen P. Increased cancer incidence after radioiodine treatment for hyperthyroidism. Cancer. 2007;109(10):1972-9.

14. Franklyn JA, Maisonneuve P, Sheppard M, Betteridge J, Boyle P. Cancer incidence and mortality after radioiodine treatment for hyperthyroidism: a population-based cohort study. Lancet. 1999;353(9170):2111-5.

15. Krause S, Dinkelborg LM. Intratumoral radiotherapy with targeted radiopharmaceuticals is superior to that with non-targeted tracers. Eur J Nucl Med Mol Imaging. 2005;32(1 Suppl):S207.

16. Krenning EP, Kwekkeboom DJ, Bakker WH, Breeman WA, Kooij PP, et al. Somatostatin receptor scintigraphy with [111In-DTPA-D-Phe1]- and [123I-Tyr3]-octreotide: the Rotterdam experience with more than 1000 patients. Eur J Nucl Med. 1993;20(8):716-31.

17. Cordier D, Forrer F, Bruchertseifer F, Morgenstern A, Apostolidis $\mathrm{C}$, Good S, et al. Targeted alpha-radionuclide therapy of functionally critically located gliomas with 213Bi-DOTA-[Thi8, Met(O2)11]-substance P: a pilot trial. Eur J Nucl Med Mol Imaging. 2010;37(7):1335-44.

18. Kneifel S, Cordier D, Good S, Ionescu MC, Ghaffari A, Hofer S, et al. Local targeting of malignant gliomas by the diffusible peptidic vector 1,4,7,10-tetraazacyclododecane-1-glutaric acid-4,7,10triacetic acid-substance p. Clin Cancer Res. 2006;12(12):384350.

19. Garin E, Lenoir L, Edeline J, Laffont S, Mesbah H, Porée P, et al. Boosted selective internal radiation therapy with 90Y-loaded glass microspheres (B-SIRT) for hepatocellular carcinoma patients: a new personalized promising concept. Eur J Nucl Med Mol Imaging. 2013. doi: 10.1007/s00259-013-2395-x.

20. Fowler JF. 21 years of biologically effective dose. Br J Radiol. 2010;83(991):554-68. 